\title{
Anomalous scaling of passively advected magnetic field in the presence of strong anisotropy
}

\author{
M. Hnatich ${ }^{1}$, J. Honkonen ${ }^{2}$, M. Jurcisin ${ }^{1,3}$, A. Mazzino ${ }^{4}$, and S. Sprinc ${ }^{1}$ \\ 1 Institute of Experimental Physics, Slovak Academy of Sciences, Watsonova 47, 04353 Košice, Slovakia \\ 2 Department of Physical Sciences, Gustav Hällströmin katu 2, \\ 00014 University of Helsinki and National Defence College, P.0. Box 7, 00861 Helsinki, Finland \\ 3 Bogolyubov Laboratory of Theoretical Physics, Joint Institute for Nuclear Research, \\ 141980 Dubna, Moscow Region, Russian Federation \\ 4 INFM-Department of Physics, University of Genova, I-16146 Genova, Italy
}

\begin{abstract}
Inertial-range scaling behavior of high-order (up to order $N=51$ ) structure functions of a passively advected vector field has been analyzed in the framework of the rapid-change model with strong small-scale anisotropy with the aid of the renormalization group and the operator-product expansion. It has been shown that in inertial range the leading terms of the structure functions are coordinate independent, but powerlike corrections appear with the same anomalous scaling exponents as for the passively advected scalar field. These exponents depend on anisotropy parameters in such a way that a specific hierarchy related to the degree of anisotropy is observed. Deviations from power-law behavior like oscillations or logarithmic behavior in the corrections to structure functions have not been found.
\end{abstract}

PACS numbers: PACS numbers: 47.10.+g, 47.27.-i, 05.10.Cc

\section{INTRODUCTION}

Justification of the basic principles of the Kolmogorov-Obukhov (KO) phenomenological theory [1, 2, 3] and the investigation of possible deviations from its conclusions within the framework of a microscopic model is one of the main tasks in the theory of the fully developed turbulence and related models, e.g. stochastic magnetohydrodynamics (MHD).

According to the KO theory, the following single-time structure functions in the inertial range $\left(r_{d} \ll r \ll r_{l}\right)$

$$
S_{N}(r) \equiv\left\langle\left[v_{r}(\mathbf{x}, t)-v_{r}\left(\mathbf{x}^{\prime}, t\right)\right]^{N}\right\rangle, \quad r=\left|\mathbf{x}-\mathbf{x}^{\prime}\right|
$$

are independent of both the external (integral) scale $r_{l}$ and internal (viscous) scale $r_{d}$, the latter being tantamount to independence of viscosity. These requirements are the famous first and second hypotheses of Kolmogorov, respectively. In Eq. (11) $v_{r}$ denotes the component of the velocity field directed along the separation vector $\mathbf{r}=\mathbf{x}-\mathbf{x}^{\prime}$. Dimensional arguments then determine the scale-invariant form of the structure functions (1) as

$$
S_{N}(r)=\text { const } \times(\bar{\epsilon} r)^{N / 3},
$$

where $\bar{\epsilon}$ is the mean dissipation rate [1, 2, 3].

On the other hand, both theoretical and experimental results reveal some deviations from the KO theory [1, 4], viz. contradiction with the first Kolmogorov hypothesis. For the structure functions (11) it means that they have to be modified in the following way

$$
S_{N}(r)=(\bar{\epsilon} r)^{N / 3} \zeta_{N}\left(r / r_{l}\right),
$$

where $\zeta_{N}\left(r / r_{l}\right)$ are scaling functions with powerlike behavior in the asymptotic region $r / r_{l} \ll 1$

$$
\zeta_{N}\left(r / r_{l}\right) \simeq \text { const } \times\left(\frac{r}{r_{l}}\right)^{q_{N}}
$$

The singular dependence of the structure functions on $r_{l}$ in the limit $r_{l} \rightarrow \infty$ together with nonlinearity of the exponents $q_{N}$ as functions of $N$ is called "anomalous scaling". Theoretical explanation of such behavior is based on strongly developed fluctuations of the dissipation rate, i.e. intermittency [1, 3].

A suitable and also powerful method of studying self-similar scaling behavior is that of the renormalization group (RG) [5, 6, 7, 8]. In the theory of critical phenomena it successfully explains the origin of the critical scaling. This technique is also applicable to the theory of turbulence, see Refs. [6, [8, [9] and references therein.

The traditional approach to the description of fully developed turbulence is based on the stochastic Navier-Stokes equation [10]. Within the RG method the second Kolmogorov hypothesis (independence of the viscous length $r_{d}$ ) 
was proved for structure functions (11) for a variety of realistic random forces, (see Ref. [8]). This fact leads to the existence of the IR scaling $\left(r \gg r_{d}\right)$ with definite "critical dimensions"

$$
\Delta\left[S_{N}\right]=-\frac{N}{3}
$$

with the customary convention $\Delta[r]=-1$ for scaling dimensions. In the framework of the RG approach, critical dimensions like (4) arise as coefficients in the differential RG equations. On the other hand, it is not possible to infer the form of the scaling functions $\zeta_{N}\left(r / r_{l}\right)$ in Eq. (2) from the RG equations.

The standard way to investigate the dependence of the scaling functions on the argument $r / r_{l}$ in the limit case $r / r_{l} \rightarrow 0$ is the utilization of the operator-product expansion (OPE), see Refs. [5, 6, 8]. The OPE leads to the following representation of scaling functions (3)

$$
\zeta_{N}\left(r / r_{l}\right)=\sum_{F} C_{F}\left(\frac{r}{r_{l}}\right)^{\Delta_{F}}, r / r_{l} \rightarrow 0,
$$

where summation over all possible composite operators $F$ (i.e. products of fields and their derivatives) is implied (for details, see Sec. IV], $\Delta_{F}$ are their critical dimensions, and coefficients $C_{F}$ are regular functions of $r_{l}^{-1}$.

Contrary to the theory of critical phenomena, where there are physical reasons to believe that all relevant composite operators have positive critical dimensions $\left(\Delta_{F}>0\right)$ for physical values of parameters [this is why the leading term in the expansion (5) is given by the trivial operator $F=1\left(\Delta_{1}=0\right)$ in that case], in the theory of fully developed turbulence based on the stochastic Navier-Stokes equation critical dimensions of many composite operators are definitely negative for physical values of parameters. Existence of these "dangerous operators" leads to singular behavior of structure functions in the limit $r / r_{l} \rightarrow 0$ [8]. In the stochastic Navier-Stokes model dangerous operators enter into the OPE in the form of infinite families with the spectrum of critical dimensions unbounded from below, and a nontrivial problem of the summation of their contributions arises. This is an unsolved problem of the theory.

In a situation where there are difficulties to study the anomalous scaling in the stochastic Navier-Stokes model (this applies to the stochastic MHD as well) it does not seem to be unreasonable to consider simpler models, which have features similar to real turbulent flow, and, on the other hand, are easier for investigation. An important role in this study was played by the model of passive advection of a scalar quantity (temperature or concentration of the tracer) by a uncorrelated-in-time Gaussian velocity field [11]. Models of passively advected vector fields [12] are straightforward generalizations of the model of the passive advection of a scalar field.

In the present work the spatial structure of correlations of fluctuations of the magnetic (vector) field $\mathbf{b}$ in a given turbulent fluid in the framework of the kinematic MHD Kazantsev-Kraichnan model (KMHD) is studied. These fluctuations are generated stochastically by a Gaussian random emf and a white in time and anisotropic self-similar in space Gaussian drift. The main goal is the calculation of the anomalous exponents as functions of the anisotropy parameters of the drift. From the mathematical point of view the present model is found to be similar to the model of a passive scalar quantity advected by a Gaussian strongly anisotropic velocity field [13] in that the fluctuation contributions to the critical dimensions $\Delta_{F}$ of the OPE representation (5) of the structure functions coincide in both cases and thus the hierarchical dependence on the degree of anisotropy is also the same. Here, numerical calculation of the critical dimensions $\Delta_{F}$ in the one-loop approximation has been extended to dimensions related to structure functions of order $N=51$ to explore possible departures from powerlike asymptotic behavior. However, contrary to the scalar case, in the inertial range the leading terms of the structure functions of the magnetic field themselves are shown to be coordinate independent with powerlike corrections whose exponents are generated by the calculated critical dimensions.

\section{KINEMATIC MHD KAZANTSEV-KRAICHNAN MODEL}

Consider passive advection of a solenoidal magnetic field $\mathbf{b} \equiv \mathbf{b}(\mathbf{x}, t)$ in the framework of the KMHD model described by the stochastic equation

$$
\partial_{t} \mathbf{b}=\nu_{0} \triangle \mathbf{b}-(\mathbf{v} \cdot \nabla) \mathbf{b}+(\mathbf{b} \cdot \nabla) \mathbf{v}+\mathbf{f}
$$

where $\partial_{t} \equiv \partial / \partial t, \triangle \equiv \nabla^{2}$ is the Laplace operator, $\nu_{0}$ is the coefficient of the magnetic diffusivity, and $\mathbf{v} \equiv \mathbf{v}(\mathbf{x}, t)$ is a random solenoidal (owing to the incompressibility) velocity field. Thus, both $\mathbf{v}$ and $\mathbf{b}$ are divergence-free vector fields: $\nabla \cdot \mathbf{v}=\nabla \cdot \mathbf{b}=0$. A transverse Gaussian emf flux density $\mathbf{f} \equiv \mathbf{f}(\mathbf{x}, t)$ with zero mean and the correlation function

$$
D_{i j}^{f} \equiv\left\langle f_{i}(\mathbf{x}, t) f_{j}\left(\mathbf{x}^{\prime}, t^{\prime}\right)\right\rangle=\delta\left(t-t^{\prime}\right) C_{i j}(\mathbf{r} / L), \quad \mathbf{r}=\mathbf{x}-\mathbf{x}^{\prime}
$$


is the source of the fluctuations of the magnetic field $\mathbf{b}$. The parameter $L$ represents an integral scale related to the stirring, and $C_{i j}$ is a function finite in the limit $L \rightarrow \infty$. In the present treatment its precise form is irrelevant, and with no loss of generality, we take $C_{i j}(0)=1$ in what follows. The random velocity field $\mathbf{v}$ obeys Gaussian statistics with zero mean and the correlation function

$$
D_{i j}^{v}(\mathbf{x}, t) \equiv\left\langle v_{i}(\mathbf{x}, t) v_{j}(0,0)\right\rangle=\frac{D_{0} \delta(t)}{(2 \pi)^{d}} \int d^{d} \mathbf{k} \frac{e^{i \mathbf{k} \cdot \mathbf{x}} T_{i j}(\mathbf{k})}{\left(k^{2}+r_{l}^{-2}\right)^{d / 2+\epsilon / 2}},
$$

where $r_{l}$ is another integral scale. In general, the scale $r_{l}$ may be different from the integral scale $L$, below we, however, take $r_{l} \simeq L . D_{0}>0$ is an amplitude factor related to the coupling constant $g_{0}$ of the model by the relation $D_{0} / \nu_{0} \equiv g_{0} \equiv \Lambda^{\epsilon}$, where $\Lambda$ is the characteristic UV momentum scale, and $0<\epsilon<2$ is a free parameter. Its "physical" value $\epsilon=4 / 3$ corresponds to the Kolmogorov scaling of the velocity correlation function in developed turbulence. $d$ is the dimensionality of the coordinate space. In the isotropic case, the second-rank tensor $T_{i j}(\mathbf{k})$ in Eq. (8) has the simple form of the ordinary transverse projector: $T_{i j}(\mathbf{k})=P_{i j}(\mathbf{k}) \equiv \delta_{i j}-k_{i} k_{j} / k^{2}$.

In what follows we will be interested in the asymptotic behavior of the structure functions $S_{N}(r)$ within the inertial range [defined by the inequalities $r_{d} \ll r \ll r_{l}$, where $r_{d} \simeq \Lambda^{-1}$ is an internal (viscous) scale], which represent the equal-time correlations of the $N$ th power of the projection of the field $\mathbf{b}$ onto the direction along the separation vector of two different space points $\mathbf{x}$ and $\mathbf{x}^{\prime}$

$$
S_{N}(r) \equiv\left\langle\left[b_{r}(\mathbf{x}, t)-b_{r}\left(\mathbf{x}^{\prime}, t\right)\right]^{N}\right\rangle, \quad r \equiv\left|\mathbf{x}-\mathbf{x}^{\prime}\right| .
$$

Dimensional analysis yields

$$
S_{N}(r)=\nu_{0}^{-N / 2} r^{N} R_{N}\left(r / r_{d}, r / r_{l}\right),
$$

where $R_{N}$ are functions of dimensionless parameters. When the random source field $\mathbf{f}$ and the velocity field $\mathbf{v}$ are uncorrelated, the odd functions $S_{2 n+1}$ vanish, however. The standard perturbation expansion (series in $g_{0}$ ) is ill suited for calculation of structure functions (10) in the limit $r / r_{d} \rightarrow \infty$ and $r / r_{l} \rightarrow 0$, due to the singular behavior of the coefficients of the expansion. Therefore, to find the correct IR behavior it is necessary to sum the whole series. Such a summation can be carried out within the field-theoretic RG and OPE. A compact description of this procedure is presented in Refs. 14, 15, 16] (see also Ref. 13]). Below we remind basic ideas and results referring to the isotropic case for simplicity of notation.

The RG analysis can be divided into two main parts. First, the UV renormalization of structure functions (9) is carried out. As a consequence of this the asymptotic behavior of functions like (9) for $r / r_{d} \gg 1$ and arbitrary but fixed $r / r_{l}$ is given by IR stable fixed point(s) of the corresponding RG equations and for functions (9) the following asymptotic form is obtained

$$
S_{N}(r) \sim \nu_{0}^{-N / 2} r^{N}\left(\frac{r}{r_{d}}\right)^{-\gamma_{N}^{*}} \xi_{N}\left(r / r_{l}\right), \quad r / r_{d} \gg 1 .
$$

where the scaling functions $\xi_{N}\left(r / r_{l}\right)$ remain unknown. In the standard language of the theory of critical phenomena (see for example Ref. [5, 6] ), the critical dimensions $\Delta\left[S_{N}\right]$ of the functions $S_{N}$ are given by the relations $\Delta\left[S_{N}\right]=$ $-N+\gamma_{N}^{*}$, where $\gamma_{N}^{*}$ are "anomalous dimensions". The dimensions $\Delta\left[S_{N}\right]$ are calculated as series in $\epsilon$, and this is why the exponent $\epsilon$ here plays the role analogous to the parameter $4-d$ in the RG theory of critical phenomena. Another parallel is related to the parameter $r_{l}$ which is an analog of the correlation length $r_{c},[5,6]$.

Second, the small $r / r_{l}$ behavior of the functions $\xi_{N}\left(r / r_{l}\right)$ has to be estimated. This may be done using the OPE, which leads to the following asymptotic form in the limit $r / r_{l} \rightarrow 0$

$$
\xi_{N}\left(r / r_{l}\right)=\sum_{F} C_{F}\left(r / r_{l}\right)\left(\frac{r}{r_{l}}\right)^{\Delta_{F}}
$$

where $C_{F}\left(r / r_{l}\right)$ are coefficients regular in $r / r_{l}$. The summation is implied over all possible renormalized scale-invariant composite operators $F$, and $\Delta_{F}$ are their critical dimensions.

In the limit $L / r \rightarrow \infty$ correlation function (7) of the random source field is uniform in space, which - as usual in stochastic models describing turbulence [7, 8, 13, 14, 15, 16] - brings about composite operators with negative critical dimensions (dangerous composite operators) in the asymptotic analysis. Contributions of these dangerous operators to the OPE imply singular behavior of the scaling functions in the limit $r / r_{l} \rightarrow 0$. The leading term is given by the operator with the most negative critical dimension $\Delta_{F}$. In our model the leading contributions to 
even structure functions $S_{N}$ are given by scalar operators $F_{N}=\left(b_{i} b_{i}\right)^{N / 2}$ with their critical dimensions $\Delta_{N}$, which eventually determine the asymptotic behavior of the structure functions $S_{N}$ of the form

$$
S_{N}(r) \propto \nu_{0}^{-N / 2} r^{N}\left(\frac{r}{r_{d}}\right)^{-\gamma_{N}^{*}}\left(\frac{r}{r_{l}}\right)^{\Delta_{N}}=\nu_{0}^{-N / 2} r_{l}^{-\Delta_{N}} r_{d}^{\gamma_{N}^{*}}
$$

where $\Delta_{N}=-N+\gamma_{N}^{*}$. Calculation shows that the anomalous dimensions $\gamma_{N}^{*}$ as well as the critical dimensions $\Delta_{N}$ in the model considered in the one-loop approximation are related to critical dimensions of composite operators of a simpler model of passively advected scalar field [14], viz. $\gamma_{N}^{*}$ are given by

$$
\gamma_{N}^{*}=\frac{N \epsilon}{2}-\frac{N(N / 2-1) \epsilon}{(d+2)}+O\left(\epsilon^{2}\right) .
$$

From relation (11) it follows that in the inertial range the structure functions are flat: $S_{N}(r) \sim$ const! Taking into account the nonlinear dependence on $N$ of the anomalous dimensions (12) we see that the deviation from the Kolmogorov scaling is extremely large in this case. It should be emphasized that this conclusion persists to all orders in perturbation theory and is not an artefact of the present one-loop calculation. Below it will be shown that these relations are stable against small-scale anisotropy.

In the anisotropic case we will assume that the statistics of the velocity field is anisotropic at all scales and replace the ordinary transverse projection operator in Eq. (8) with the operator

$$
T_{i j}(\mathbf{k})=\left(1+\alpha_{10} \frac{\mathbf{n} \cdot \mathbf{k}}{k^{2}}\right) P_{i j}(\mathbf{k})+\alpha_{20} n_{s} n_{l} P_{i s}(\mathbf{k}) P_{j l}(\mathbf{k}),
$$

where $P_{i j}(\mathbf{k})$ is the usual transverse projection operator, the unit vector $\mathbf{n}$ determines the distinguished direction, and $\alpha_{10}, \alpha_{20}$ are parameters characterizing the anisotropy. The positive definiteness of the correlation function (8) imposes the following restrictions on their values: $\alpha_{10}, \alpha_{20}>-1$. The operator (13) is a special case of the general transverse structure that possesses uniaxial anisotropy:

$$
T_{i j}(\mathbf{k})=a(\psi) P_{i j}(\mathbf{k})+b(\psi) n_{s} n_{l} P_{i s}(\mathbf{k}) P_{j l}(\mathbf{k}),
$$

where $\psi$ denotes the angle between the vectors $\mathbf{n}$ and $\mathbf{k}(\mathbf{n} \cdot \mathbf{k}=k \cos \psi)$. Using Gegenbauer polynomials [17] the scalar functions in representation (14) may be expressed in the form

$$
a(\psi)=\sum_{i=0}^{\infty} a_{i} P_{2 i}(\cos \psi), \quad b(\psi)=\sum_{i=0}^{\infty} b_{i} P_{2 i}(\cos \psi) .
$$

For the case of passively advected scalar, it was shown in Ref. 13] that all main features of the general model with the anisotropy structure represented by Eq. (14) are included in the simplified model with the special form of the transverse operator given by Eq. (13). The same argument applies for the present case of passively advected vector field as well.

The uniaxial anisotropy projector (13) has already been widely used in analyzes of the anisotropically driven Navier-Stokes equation, MHD turbulence equations and passive advection equations [21]. However, these studies were limited to the investigation of the existence and stability of the fixed points with the subsequent calculation of the critical dimensions of the basic quantities leaving the calculation of the anomalous exponents in those models an open problem.

The strong small-scale anisotropy (14) does not change the leading inertial-range term of the structure functions (11), but the anisotropy shows in the corrections to it. Indeed, combining the results of multiplicative renormalization and OPE in the manner sketched above for the isotropic case, we arrive at the conclusion that the inertial-range asymptotics of the structure functions of the passively advected vector field $S_{N}$ is a constant independent of $r$ with growing powerlike corrections effected by the small-scale anisotropy:

$$
S_{N}(r) \sim D_{0}^{-N / 2} r_{d}^{N-N \epsilon / 2}\left(\frac{r_{d}}{r_{l}}\right)^{\Delta_{N}}\left[c_{N}+\sum_{p=1}^{N} c_{N, p}\left(\frac{r}{r_{l}}\right)^{\Delta_{[N, p]}-\Delta_{N}}+\sum_{\substack{M+K=N \\ p \leq M, q \leq K}} c_{M, p, q}\left(\frac{r}{r_{l}}\right)^{\Delta_{[M, p]}+\Delta_{[K, q]}-\Delta_{N}}\right],
$$

where the critical dimensions $\Delta_{N}$ are given by Eq. (12) at the order $O(\epsilon)$, whereas the critical dimensions $\Delta_{[M, p]}$ will be defined and calculated below [Eq. [40] ]. This is similar to the asymptotic behavior of the structure functions of the passive scalar advected by a compressible vector field [15]. 


\section{FIELD-THEORETIC FORMULATION, RENORMALIZATION, AND RG ANALYSIS}

The stochastic problem (6)-(8) is equivalent to the field-theoretic model of the set of the three fields $\Phi=\left\{\mathbf{b}^{\prime}, \mathbf{b}, \mathbf{v}\right\}$ with the action functional

$$
S(\Phi) \equiv \frac{1}{2} \mathbf{b}^{\prime} D_{b} \mathbf{b}^{\prime}+\mathbf{b}^{\prime}\left[-\partial_{t}-(\mathbf{v} \cdot \nabla)+\nu_{0} \Delta\right] \mathbf{b}+\mathbf{b}^{\prime}(\mathbf{b} \cdot \nabla) \mathbf{v}-\frac{1}{2} \mathbf{v} D_{v}^{-1} \mathbf{v},
$$

where $\mathbf{b}^{\prime}$ is an auxiliary field (all required integrations over space-time coordinates and summations over the vector indices are implied). The first five terms in Eq. (15) represent the De Dominicis-Janssen action corresponding to the stochastic problem at fixed $\mathbf{v}$ (see, e.g., Refs. 22]), whereas the last term represents the Gaussian averaging over $\mathbf{v}$. $D_{b}$ and $D_{v}$ are the correlation functions (7) and (8), respectively.

In this field-theoretic language, the structure functions (91) are defined as

$$
S_{N}(r) \equiv \int \mathcal{D} \Phi\left[b_{r}(\mathbf{x}, t)-b_{r}\left(\mathbf{x}^{\prime}, t\right)\right]^{N} e^{S(\Phi)}
$$

with the action $S(\Phi)$ defined above.

Action (15) is given in a form convenient for application of the quantum-field perturbation analysis with the standard Feynman-diagram technique. The quadratic part of the action determines the matrix of bare propagators. For the fields $\mathbf{b}^{\prime}$ and $\mathbf{b}$ the propagators in the wave-vector-frequency representation are

$$
\begin{aligned}
\left\langle b_{i} b_{j}^{\prime}\right\rangle_{0} & =\left\langle b_{j}^{\prime} b_{i}\right\rangle_{0}^{*}=\frac{P_{i j}(\mathbf{k})}{-i \omega+\nu_{0} k^{2}}, \\
\left\langle b_{i} b_{j}\right\rangle_{0} & =\frac{C_{i j}(\mathbf{k})}{\omega^{2}+\nu_{0}^{2} k^{4}}, \\
\left\langle b_{i}^{\prime} b_{j}^{\prime}\right\rangle_{0} & =0
\end{aligned}
$$

where $C_{i j}(\mathbf{k})$ is the Fourier transform of the function $C_{i j}(\mathbf{r} / L)$ from Eq. (7). The bare propagator of the velocity field $\langle\mathbf{v v}\rangle_{0} \equiv\langle\mathbf{v v}\rangle$ is defined by Eq. (8) with the transverse projector given by Eq. (13). The interaction in the model is given by the nonlinear terms $-b_{i}^{\prime}(\mathbf{v} \cdot \nabla) b_{i}+b_{i}^{\prime}(\mathbf{b} \cdot \nabla) v_{i} \equiv b_{i}^{\prime} V_{i j l} v_{j} b_{l}$ with the vertex factor which in the wave-number-frequency representation has the following form

$$
V_{i j l}=i\left(\delta_{i j} k_{l}-\delta_{i l} k_{j}\right)
$$

With the use of the standard power counting [5, 6] (see also Ref. [13] for peculiarities of rapid-change passive advection models) correlation functions with superficial UV divergences may be identified. These are correlation functions containing frequency-wave-vector integrals divergent in the limit $\epsilon \rightarrow 0$ with divergences brought about by integration over large wave numbers and correspondingly having non-negative wave-number dimension. In the present model superficial divergences exist only in the one-particle-irreducible (1PI) Green function $\Gamma_{\mathbf{b}^{\prime} \mathbf{b}}$. In the isotropic case this Green function gives rise only to the renormalization of the term $\nu_{0} \mathbf{b}^{\prime} \Delta \mathbf{b}$ of action (15) and the corresponding UV divergences may be fully absorbed in the proper redefinition of the existing parameters $g_{0}, \nu_{0}$ so that all correlation functions calculated in terms of the renormalized parameters $g$ and $\nu$ are UV finite.

When anisotropy is introduced, however, the situation becomes more complicated, because the 1PI Green function $\Gamma_{\mathbf{b}^{\prime} \mathbf{b}}$ produces divergences corresponding to the structure $\mathbf{b}^{\prime}(\mathbf{n} \nabla)^{2} \mathbf{b}$ in the action of the model [due to peculiarities of the rapid-change models $\left[14\right.$ the term $\left(\mathbf{b}^{\prime} \mathbf{n}\right) \triangle(\mathbf{b n})$ possible on dimensional and symmetry grounds does not appear]. The term $\mathbf{b}^{\prime}(\mathbf{n} \nabla)^{2} \mathbf{b}$ is not present in the original unrenormalized action (15), but has to be added to the renormalized action, therefore the model is not multiplicatively renormalizable. In such a case it is customary to extend the original action (15) by including all terms needed for the renormalization of the correlation functions and thus adding new parameters. As a result the extended model is described by a new action of the form:

$$
S(\Phi) \equiv \frac{1}{2} \mathbf{b}^{\prime} D_{b} \mathbf{b}^{\prime}+\mathbf{b}^{\prime}\left[-\partial_{t}-(\mathbf{v} \cdot \nabla)+\nu_{0} \Delta+\chi_{0} \nu_{0}(\mathbf{n} \cdot \nabla)^{2}\right] \mathbf{b}+\mathbf{b}^{\prime}(\mathbf{b} \cdot \nabla) \mathbf{v}-\frac{1}{2} \mathbf{v} D_{v}^{-1} \mathbf{v},
$$

where a new unrenormalized parameter $\chi_{0}$ has been introduced.

Of course, the bare propagators (17) of the isotropic model are modified and for the extended action (18) assume the form

$$
\begin{aligned}
\left\langle b_{i} b_{j}^{\prime}\right\rangle_{0} & =\left\langle b_{j}^{\prime} b_{i}\right\rangle_{0}^{*}=\frac{P_{i j}(\mathbf{k})}{-i \omega+\nu_{0} k^{2}+\chi_{0} \nu_{0}(\mathbf{n} \cdot \mathbf{k})^{2}}, \\
\left\langle b_{i} b_{j}\right\rangle_{0} & =\frac{C_{i j}(\mathbf{k})}{\left|-i \omega+\nu_{0} k^{2}+\chi_{0} \nu_{0}(\mathbf{n} \cdot \mathbf{k})^{2}\right|^{2}}, \\
\left\langle b_{i}^{\prime} b_{j}^{\prime}\right\rangle_{0} & =0 .
\end{aligned}
$$




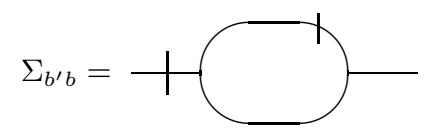

FIG. 1: The (exact) graphical expression for the self-energy operator $\Sigma_{b^{\prime} b}$ of the response function of the passive vector field. The plain line denotes the bare propagator (20), and the line with slash (denoting the end corresponding to the arguments of the field $\mathbf{b}^{\prime}$ ) corresponds to the bare propagator (19).

After this modification all terms needed to remove the divergences are present in action (18), therefore the model becomes multiplicatively renormalizable allowing for the standard RG analysis. The corresponding renormalized action may be written down immediately:

$$
S_{R}(\Phi) \equiv \frac{1}{2} \mathbf{b}^{\prime} D_{b} \mathbf{b}^{\prime}+\mathbf{b}^{\prime}\left[-\partial_{t}-(\mathbf{v} \cdot \nabla)+\nu Z_{1} \Delta+\chi \nu Z_{2}(\mathbf{n} \cdot \nabla)^{2}\right] \mathbf{b}+\mathbf{b}^{\prime}(\mathbf{b} \cdot \nabla) \mathbf{v}-\frac{1}{2} \mathbf{v} D_{v}^{-1} \mathbf{v} .
$$

Here, $Z_{1}$ and $Z_{2}$ are the renormalization constants in which the UV divergent parts of the 1PI response function $\Gamma_{\mathbf{b}^{\prime} \mathbf{b}}$ are absorbed. The renormalized action (21) leads to the multiplicative renormalization of the parameters $\nu_{0}, g_{0}$ and $\chi_{0}$ :

$$
\nu_{0}=\nu Z_{\nu}, \quad g_{0}=g \mu^{\epsilon} Z_{g}, \quad \chi_{0}=\chi Z_{\chi},
$$

where $\nu, g$, and $\chi$ are renormalized counterparts of the bare parameters, and $\mu$ is a scale-setting parameter with the same canonical dimension as the wave number. The anisotropy parameters $\alpha_{01}$ and $\alpha_{02}$ are not renormalized, therefore their renormalized counterparts $\alpha_{1}$ and $\alpha_{2}$ may be put equal to the unrenormalized parameters. In what follows, we will work in the minimal subtraction (MS) scheme, in which - in the one-loop aproximation - renormalization constants have the form $1+A / \epsilon$, where the amplitude $A$ is a function of $g, \chi, \alpha_{1}, \alpha_{2}$, and $d$, but independent of $\epsilon$.

Identification of the unrenormalized action (18) with the renormalized one (21) leads to the following relations between the renormalization constants:

$$
Z_{1}=Z_{\nu}, \quad Z_{2}=Z_{\chi} Z_{\nu}, \quad Z_{g}=Z_{\nu}^{-1}
$$

It has to be mentioned that the rapid-change models like (18) have the nice feature that in all multiloop diagrams of the self-energy operator $\Sigma_{b^{\prime} b}$ closed circuits of the retarded bare propagators $\left\langle\mathbf{b b}^{\prime}\right\rangle_{0}$ are produced, because the propagator $\langle\mathbf{v v}\rangle_{0}$ is proportional to the $\delta$ function in time. As a result, the one-loop self energy operator $\Sigma_{b^{\prime} b}$ with the graphical notation of Fig. 1 is exact.

The divergent part of the graph in Fig. 1 is

$$
\Sigma_{b^{\prime} b}(p)=-\frac{g \nu C_{d}}{2 d(d+2) \epsilon}\left\{\left[(d-1)(d+2)+\alpha_{1}(d+1)+\alpha_{2}\right] p^{2}-\left(2 \alpha_{1}-\left(d^{2}-2\right) \alpha_{2}\right)(\mathbf{n} \cdot \mathbf{p})^{2}\right\},
$$

where $C_{d} \equiv S_{d} /(2 \pi)^{2}$ and $S_{d}=2 \pi^{d / 2} / \Gamma(d / 2)$ is the area of the $d$-dimensional sphere of unit radius. The expression (22) leads to a straightforward determination of the renormalization constants $Z_{1}$ and $Z_{2}$ :

$$
\begin{aligned}
& Z_{1}=1-\frac{g C_{d}}{2 d(d+2) \epsilon}\left[(d-1)(d+2)+\alpha_{1}(d+1)+\alpha_{2}\right], \\
& Z_{2}=1-\frac{g C_{d}}{2 d(d+2) \chi \epsilon}\left[-2 \alpha_{1}+\left(d^{2}-2\right) \alpha_{2}\right] .
\end{aligned}
$$

With the proper choice of the renormalization constants this renormalization procedure gives rise to UV-finite correlation functions with separate space and time arguments. Independence of the original unrenormalized model of the scale-setting parameter $\mu$ of the renormalized model yields the RG differential equations for the renormalized correlation functions of the fields, e.g.

$$
\left(\mathcal{D}_{\mu}+\beta_{g} \partial_{g}+\beta_{\chi} \partial_{\chi}-\gamma_{\nu} \mathcal{D}_{\nu}\right)\left\langle\mathbf{b}(\mathbf{x}, t) \mathbf{b}\left(\mathbf{x}^{\prime}, t\right)\right\rangle_{R}=0
$$

with the definition $\mathcal{D}_{x} \equiv x \partial_{x}$ for any variable $x$, and with the following definition of the RG functions (the $\beta$ functions and the anomalous dimensions $\gamma$ )

$$
\gamma_{i} \equiv \widetilde{\mathcal{D}}_{\mu} \ln Z_{i}
$$

for any renormalization constant $Z_{i}$, and

$$
\beta_{g} \equiv \widetilde{\mathcal{D}}_{\mu} g=g\left(-\epsilon+\gamma_{1}\right), \quad \beta_{\chi} \equiv \widetilde{\mathcal{D}}_{\mu} \chi=\chi\left(\gamma_{1}-\gamma_{2}\right),
$$


where $\widetilde{\mathcal{D}} \equiv \mu \partial_{\mu}$ denotes derivative with fixed bare parameters of the extended action (18).

For the anomalous dimensions $\gamma_{1}$ and $\gamma_{2}$ we obtain from Eq. (23)

$$
\begin{aligned}
& \gamma_{1}=\frac{g C_{d}}{2 d(d+2)}\left[(d-1)(d+2)+\alpha_{1}(d+1)+\alpha_{2}\right], \\
& \gamma_{2}=1-\frac{g C_{d}}{2 d(d+2) \chi}\left[-2 \alpha_{1}+\left(d^{2}-2\right) \alpha_{2}\right] .
\end{aligned}
$$

It should be emphasized that both the renormalization constants (23) and the corresponding anomalous dimensions (25) and (26) in the present model are exact, i.e., they have no corrections of order $g^{2}$ or higher.

The fixed points $\left(g_{*}, \chi_{*}\right)$ of the RG equations are defined by the system of two equations

$$
\beta_{g}\left(g_{*}, \chi_{*}\right)=0, \quad \beta_{\chi}\left(g_{*}, \chi_{*}\right)=0 .
$$

The IR stability of a fixed point is determined by the condition that real parts of the eigenvalues of the matrix

$$
\omega=\left(\begin{array}{cc}
\partial_{g} \beta_{g} & \partial_{\chi} \beta_{g} \\
\partial_{g} \beta_{\chi} & \partial_{\chi} \beta_{\chi}
\end{array}\right)_{\substack{g=g_{*} \\
\chi=\chi_{*}}}
$$

are positive. Calculation shows that the RG equations have only one non-trivial IR stable fixed point defined by expressions

$$
\begin{aligned}
g_{*} & =\frac{2 d(d+2) \epsilon}{C_{d}\left[(d-1)(d+2)+\alpha_{1}(d+1)+\alpha_{2}\right]}, \\
\chi_{*} & =\frac{-2 \alpha_{1}+\left(d^{2}-2\right) \alpha_{2}}{(d-1)(d+2)+\alpha_{1}(d+1)+\alpha_{2}} .
\end{aligned}
$$

Both eigenvalues of the stability matrix $\omega$ are equal to $\epsilon$ at this fixed point, therefore, the IR fixed point (27), (28) is stable for $\epsilon>0$ and all values of the anisotropy parameters $\alpha_{1}$ and $\alpha_{2}$.

Rather unexpectedly, the $\beta$ functions and, consequently, the fixed points of the present model of passively advected vector field are exactly the same as in the model of passively advected scalar field [13]. In Sec. [V] it will be shown that this similarity is extended to the anomalous scaling dimensions of the composite operators in the OPE representation of the structure functions as well.

The fixed point (27), (28) governs the behavior of solutions of Eqs. (24) and the like, and at large scales far from viscous length $r \gg r_{d}$ at any fixed ratio $r / r_{l}$ yields the scaling form

$$
\langle\mathbf{b}(\mathbf{x}, t) \mathbf{b}(\mathbf{x}-\mathbf{r}, t)\rangle=D_{0}^{-1} r^{2-\epsilon} \xi_{2}\left(r / r_{l}\right),
$$

for the unrenormalized correlation function (we remind that due to the absence of field renormalization renormalized and unrenormalized correlation functions are equal but expressed in terms of different variables). It should be noted, however, that the scaling function $\xi_{2}\left(r / r_{l}\right)$ in Eq. (29) is not determined by the RG Eqs. (24).

This, however, is not enough to find the asymptotic scaling behavior of the structure functions (16), because they are linear combinations of correlation functions with coinciding arguments. The latter contain UV divergences additional to those included in the renormalization constants (23). These additional divergences due to composite operators (products of fields and their derivatives with coinciding space and time arguments) can be dealt with in a manner similar to that applied to the divergences in the usual correlation functions 6 .

\section{RENORMALIZATION AND CRITICAL DIMENSIONS OF COMPOSITE OPERATORS}

A composite operator is any product of fields and their derivatives at a single space-time point $x \equiv(\mathbf{x}, t)$, e.g. $\left[\mathbf{b}^{2}(x)\right]^{N}$ and $\left[\partial_{i} b_{j}(x) \partial_{i} b_{j}(x)\right]^{N}$. Structure functions (16) contain products of composite operators at two separate space points. These composite operators are integer powers of the field $b_{r}$. Usually, the aim of renormalization of composite operators is to make UV finite all 1PI correlation functions with the insertion of a composite operator $F$, i.e. quantities of the form

$$
\left\langle F \phi_{i_{1}}\left(\mathbf{x}_{1}, t_{1}\right) \cdots \phi_{i_{m}}\left(\mathbf{x}_{m}, t_{m}\right)\right\rangle_{1 \mathrm{PI}}
$$


Correlation functions with such insertions contain additional UV divergences, which also may be removed by a suitable renormalization procedure [5, 6]. Composite operators mix in renormalization, therefore an UV finite renormalized operator $F^{R}$ has the form $F^{R}=F+\sum \Delta F$, where the counterterms $\sum \Delta F$ are a linear combination of the operator $F$ itself and, in general, other unrenormalized operators required to make all correlation functions - generated by the renormalized action - with the insertion of $F^{R}$ UV finite. In this case homogeneous RG equations of the form (24) may be obtained for certain linear combinations of renormalized correlation functions with composite-operator insertions. Such linear combinations (basis operators $\mathcal{F}$, see below) exhibit IR scaling with definite critical dimensions $\Delta_{\mathcal{F}}$, whereas an arbitrary renormalized composite operator may be expressed as a linear combination of these basis operators.

The general procedure is the following [ [5, 6, 7, 8, 13, 14]: If $\left\{F_{\alpha}\right\}$ is a closed set of composite operators (i.e., they are mixed only with each other in renormalization), then the sets of renormalized and unrenormalized operators are related through the matrix transformation:

$$
\begin{aligned}
& F_{\alpha}=\sum_{\beta} Z_{\alpha \beta} F_{\beta}^{R}, \\
& \gamma_{F}=Z_{F}^{-1} \widetilde{\mathcal{D}}_{\mu} Z_{F},
\end{aligned}
$$

where $Z_{F} \equiv\left\{Z_{\alpha \beta}\right\}$ is the renormalization matrix and $\gamma_{F} \equiv\left\{\gamma_{\alpha \beta}\right\}$ is the corresponding matrix of anomalous dimensions for this set of operators. The renormalized composite operators obey the RG differential equations

$$
\left(\mathcal{D}_{\mu}+\beta_{g} \partial_{g}+\beta_{\chi} \partial_{\chi}-\gamma_{\nu} \mathcal{D}_{\nu}\right) F_{\alpha}^{R}=-\sum_{\beta} \gamma_{\alpha \beta} F_{\beta}^{R},
$$

which give rise to the matrix of critical dimensions $\Delta_{F} \equiv\left\{\Delta_{\alpha \beta}\right\}$ of the form

$$
\Delta_{F}=d_{F}^{k}-\Delta_{t} d_{F}^{\omega}+\gamma_{F}^{*}, \quad \Delta_{t}=-2+\epsilon,
$$

where $d_{F}^{k}$ and $d_{F}^{\omega}$, respectively, are the diagonal matrices of canonical wave-number and frequency dimensions of the operators (where the diagonal elements are sums of the corresponding dimensions of the operators included in the composite operator) and $\gamma_{F}^{*}$ is the matrix of anomalous dimensions (31) at the fixed point (27), (28).

Critical dimensions of the set $F \equiv\left\{F_{\alpha}\right\}$ are given by the eigenvalues of the matrix $\Delta_{F}$. The basis operators possessing definite critical dimensions are related to the renormalized composite operators by the matrix transformation

$$
\mathcal{F}_{\alpha}=\sum_{\beta} U_{\alpha \beta} F_{\beta}^{R}
$$

where the matrix $U_{F} \equiv\left\{U_{\alpha \beta}\right\}$ is such that the transformed matrix of critical dimensions $\Delta_{\mathcal{F}}=U_{F} \Delta_{F} U_{F}^{-1}$ is diagonal.

The structure functions contain, however, quantities which correspond to insertions of two composite operators. Therefore, it would seem that we would have to consider renormalization of products of two composite operators as well, the aim being then to render UV finite all 1PI correlation functions with two insertions of composite operators. Superficially divergent correlation functions with operator insertions are identified by power counting similar to that of the basic renormalization. In the present model such a power counting shows that insertion of products of composite operators of the structure $\mathbf{b}^{m}(\mathbf{x}, t) \mathbf{b}^{n}\left(\mathbf{x}^{\prime}, t\right)$ does not bring about any new superficial divergences and it is thus sufficient to renormalize the composite operators themselves only in order to make the structure functions UV finite. Therefore, from the RG analysis of composite operators it follows - by virtue of relations (30) and (33) - that the structure function $S_{N}$ may be expressed as a functional average of a quadratic form of basis operators:

$$
S_{N}(r)=\sum_{\alpha, \beta} B_{\alpha \beta}\left\langle\mathcal{F}_{\alpha}\left(\mathbf{x}+\frac{1}{2} \mathbf{r}, t\right) \mathcal{F}_{\beta}\left(\mathbf{x}-\frac{1}{2} \mathbf{r}, t\right)\right\rangle_{R}
$$

with coefficients $B_{\alpha \beta}$ independent of spatial coordinates. Each term in expression (34) obeys the following asymptotic form in the limit $r_{d} \ll r, r \lesssim r_{l}$

$$
\left\langle\mathcal{F}_{\alpha}\left(\mathbf{x}+\frac{1}{2} \mathbf{r}, t\right) \mathcal{F}_{\beta}\left(\mathbf{x}-\frac{1}{2} \mathbf{r}, t\right)\right\rangle_{R} \sim D_{0}^{d_{\alpha}^{\omega}+d_{\beta}^{\omega}} r^{-\Delta_{\alpha}-\Delta_{\beta}} r_{d}^{\gamma_{\alpha}^{*}+\gamma_{\beta}^{*}} \Xi_{\alpha \beta}\left(\frac{r}{r_{l}}\right)
$$

with the scaling functions $\Xi_{\alpha \beta}$ still to be determined. 
The physically interesting range of scales, however, is the inertial range, specified by the inequalities $r_{d} \ll r \ll r_{l}$. The limit $r \ll r_{l}$ may be explored with the use of the OPE [5, 6] as was already discussed in Sec. [] The basic statement of the OPE theory is that the equal-time product of two renormalized composite operators can be represented in the form

$$
F_{\alpha}^{R}\left(\mathbf{x}+\frac{1}{2} \mathbf{r}, t\right) F_{\beta}^{R}\left(\mathbf{x}-\frac{1}{2} \mathbf{r}, t\right)=\sum_{\gamma} C_{\alpha \beta \gamma}(\mathbf{r}) F_{\gamma}^{R}(\mathbf{x}, t),
$$

where the functions $C_{\alpha \beta \gamma}$ are the Wilson coefficients regular in $1 / r_{l}$, and $F_{\gamma}^{R}$ are renormalized local composite operators which appear in the formal Taylor expansion with respect to $\mathbf{r}$ together with all operators that mix with them in renormalization. If these operators have additional vector indices, they are contracted with the corresponding indices of the coefficients $C_{\alpha \beta \gamma}$.

Without loss of generality we may take the expansion on the right-hand side of Eq. (36) in terms of the basis operators with definite critical dimensions $\Delta_{\mathcal{F}}$. The renormalized correlation function $\left\langle F_{\alpha}^{R} F_{\beta}^{R}\right\rangle_{R}$ is obtained by averaging Eq. (36) with the weight generated by the renormalized action, the quantities $\langle\mathcal{F}\rangle_{R}$ appear now only on the right-hand side. Their asymptotic behavior for $r / r_{l} \rightarrow 0$ is found from the corresponding RG equations and is of the form $\langle\mathcal{F}\rangle \propto r_{l}^{-\Delta_{\mathcal{F}}}$. Comparison of the expression for a given function $\left\langle F_{\alpha}^{R} F_{\beta}^{R}\right\rangle_{R}$ in terms of the IR scaling representation of correlation functions of the basis operators (35) on one hand and the OPE representation brought about by relation (36) on the other in the limit $r_{l} \rightarrow \infty$ allows to find the asymptotic form of the scaling functions $\Xi_{\alpha \beta}\left(r / r_{l}\right)$ in relation (35).

The composite operators appearing in the expression for the structure function $S_{N}$ are products of integer powers of the field $b_{r}$ of the form $b_{r}^{N-m}(\mathbf{x}, t) b_{r}^{m}\left(\mathbf{x}^{\prime}, t\right)$. Thus, at the leading order in $\mathbf{r}$ their OPE contains operators of the closed set generated by the operator $b_{r}^{N}(\mathbf{x}, t)$. Power counting and analysis of the structure of graphs shows that this set of composite operators contains only operators consisting of exactly $N$ components of the vector field $\mathbf{b}$ (i.e. no derivatives of the field components appear). Extracting the common scaling factor prescribed by the canonical dimensions (32) of these operators the basis-operator decomposition of any term of the structure function $S_{N}$ may be written as

$$
\left\langle b_{r}^{N-m}\left(\mathbf{x}+\frac{1}{2} \mathbf{r}, t\right) b_{r}^{m}\left(\mathbf{x}-\frac{1}{2} \mathbf{r}, t\right)\right\rangle \sim D_{0}^{-N / 2} r^{N(1-\epsilon / 2)} \sum_{\alpha, \beta, \gamma} A_{\alpha \beta \gamma}\left(r / r_{l}\right)\left(\frac{r}{r_{d}}\right)^{-\gamma_{\alpha}^{*}-\gamma_{\beta}^{*}}\left(\frac{r}{r_{l}}\right)^{\Delta_{\gamma}}
$$

where the coefficients $A_{\alpha \beta \gamma}\left(r / r_{l}\right)$ are regular in $\left(r / r_{l}\right)^{2}$.

The decomposition (37) reveals the inertial-range scaling form of the structure functions. The leading singular contribution in the limit $r_{l} \rightarrow \infty, r_{d} \rightarrow 0$ is given by the basis operator $\mathcal{F}_{\gamma}$ with the minimal critical dimension $\Delta_{\gamma}$ and operators $\mathcal{F}_{\alpha}$ and $\mathcal{F}_{\beta}$ with the minimal sum of anomalous dimensions $\gamma_{\alpha}^{*}+\gamma_{\beta}^{*}$. As a result, $S_{N}$ have singular power-like behavior as $r / r_{l} \rightarrow 0$ :

$$
S_{N}(r) \sim r^{N(1-\epsilon / 2)}\left(\frac{r}{r_{d}}\right)^{-\gamma_{N}^{*}}\left(\frac{r}{r_{l}}\right)^{\Delta_{N}}
$$

with the most negative exponent $\Delta_{N}$ in the basis set generated by the composite operator $b_{r}^{N}(\mathbf{x}, t)$ and the most negative sum of anomalous dimensions $\gamma_{[M, p]}^{*}+\gamma_{[K, q]}^{*}$ subject to the conditions $M+K=N, p \leq M$ and $q \leq K$.

In our case, the leading contribution to the sum (37) from the OPE (36) will be given by the tensor composite operators constructed solely of the fields $\mathbf{b}$ without derivatives: $b_{i_{1}} \ldots b_{i_{p}}\left(b_{i} b_{i}\right)^{l}$. It is useful to deal with the scalar operators obtained by contracting the tensor with the appropriate number of the anisotropy vectors $\mathbf{n}$ :

$$
F[N, p](\mathbf{x}, t) \equiv[\mathbf{n} \cdot \mathbf{b}(\mathbf{x}, t)]^{p}\left[b_{i}(\mathbf{x}, t) b_{i}(\mathbf{x}, t)\right]^{l}
$$

with $N \equiv 2 l+p$. Power counting and analysis of graphs show that composite operators (38) for given $N$ can be mixed only with each other in renormalization

$$
F[N, p]=\sum_{p^{\prime}=0}^{N} Z_{[N, p]\left[N, p^{\prime}\right]} F^{R}\left[N, p^{\prime}\right],
$$

therefore, the corresponding renormalization matrix $Z_{[N, p]\left[N^{\prime}, p^{\prime}\right]}$ is in fact block-diagonal, i.e., $Z_{[N, p]\left[N^{\prime}, p^{\prime}\right]}=0$ for $N^{\prime} \neq N$.

A detailed account of practical calculation of the matrix of the renormalization constants $Z_{[N, p]\left[N, p^{\prime}\right]}$ (which may be readily extended to investigation of all related problems) has been given in Ref. [13] for the advection of a passive 
scalar, therefore we will not describe all details of the determination of renormalization constants in the present vector model, rather we will discuss its specific features.

It turned out that not only the $\beta$ functions in the vector and scalar models coincide, but the one-loop renormalization matrices as well. This nontrivial fact stems from the similarities of the mathematical structure of both models. In the model of scalar advection [13] the composite operators $\partial_{i_{1}} \theta \ldots \partial_{i_{p}} \theta\left(\partial_{i} \theta \partial_{i} \theta\right)^{l}$ constructed solely of the scalar gradients of the scalar admixture $\theta$ are needed for calculation of the asymptotic behavior of the structure functions, whereas in our vector case the main contribution is given by composite operators constructed solely of the fields $\mathbf{b}$ without derivatives. As direct inspection of the relevant diagrams shows, the tensor structures arising upon functional averaging in both cases are in fact identical, which yields the same renormalization matrix $Z_{[N, p]\left[N, p^{\prime}\right]}$ in both models. Thus, it is not necessary to carry out complete calculations here.

However, in Ref. 13] in the expressions for the general elements of the renormalization matrix of the composite operators there are misprints (for instance, in the definition of the quantity $Q_{1}$ in Eq. (76) of Ref. [13] $H_{4}-H_{6}$ should be replaced by $\mathrm{H}_{3}-\mathrm{H}_{6}$ ), although the numerical investigation of the critical dimensions is correct. Therefore, we present here the full formulae, in a slightly different form, however.

The only nonzero elements of the matrix $Z_{[N, p]\left[N, p^{\prime}\right]}$ are

$$
\begin{aligned}
Z_{[N, p][N, p-2]} & =\frac{g C_{d}}{d^{2}-1} \frac{1}{4 \epsilon} Q_{1}, & Z_{[N, p][N, p]} & =1+\frac{g C_{d}}{d^{2}-1} \frac{1}{4 \epsilon} Q_{2}, \\
Z_{[N, p][N, p+2]} & =\frac{g C_{d}}{d^{2}-1} \frac{1}{4 \epsilon} Q_{3}, & Z_{[N, p][N, p+4]} & =\frac{g C_{d}}{d^{2}-1} \frac{1}{4 \epsilon} Q_{4},
\end{aligned}
$$

with the coefficients $Q_{i}$ defined as follows

$$
\begin{aligned}
& Q_{1}=p(p-1)(d+1)\left(H_{0}\left(1+\alpha_{2}\right)+\left(H_{4}-H_{2}\right)\left(1-2 \alpha_{1}+3 \alpha_{2}\right)+H_{6}\left(\alpha_{1}-\alpha_{2}\right)\right) \\
& Q_{2}= H_{0}\left((d-2)(N-p)^{2}-\left(1+\alpha_{2}\right)(d+1)(p-1) p\right. \\
&\left.\quad+(N-p)\left(3+d^{2}+2 d(p-1)+2 p+\alpha_{2}(d+1)(1+2 p)\right)\right) \\
&+ H_{2}\left(\left(5+3 \alpha_{2}+\alpha_{1}(d-2)-d\right)(N-p)^{2}\right. \\
& \quad+(d+1)\left(1-\alpha_{1}+d+\alpha_{2}(d+2)\right)(p-1) p-(N-p)\left(9-2 d+d^{2}+8 p(d+1)\right. \\
&\left.\left.\quad-\alpha_{1}\left(3+d^{2}+2 d(p-1)+2 p\right)+2 \alpha_{2}(4+d+5 p(d+1))\right)\right) \\
&+ H_{4}\left(-\left(3+6 \alpha_{2}+\alpha_{1}(d-5)\right)(N-p)^{2}+(d+1)\left(\alpha_{1}(d+1)-\alpha_{2}(2 d+1)-d\right)(p-1) p\right. \\
& \quad+(N-p)\left(6(1+p(d+1))+\alpha_{2}(13+d+14 p(d+1))\right. \\
&\left.\left.\quad-\alpha_{1}\left(9+d^{2}+8 p+2 d(4 p-1)\right)\right)\right) \\
&+ H_{6}\left(\alpha_{2}+\alpha_{1}\right)\left(3(N-p)^{2}+d(d+1)(p-1) p-6(N-p)(1+p(d+1))\right) \\
& Q_{3}=H_{0}(n-p)\left(-9-d^{2}+5 N-7 p-d(-2+N+p)+\alpha_{2}(d+1)(n-3(1+p))\right) \\
&+H_{2}(N-p)\left(d^{3}+d^{2}(-2+N+p)+6(6-3 N+4 p)+d(9-5 N+13 p)\right. \\
& \quad+\alpha_{2}\left(27-13 N+21 p+d^{2}(1+2 p)+d(16-7 N+17 p)\right) \\
&\left.\quad+\alpha_{1}\left(9+d^{2}-5 N+7 p+d(-2+N+p)\right)\right) \\
&+H_{4}(N-p)(-2(2+d)(6-3 N+(4+d) p) \\
& \quad+\alpha_{1}\left(d^{3}+d^{2}(-2+N+p)+6(6-3 N+4 p)+d(9-5 N+13 p)\right) \\
&\left.\quad+\alpha_{2}\left(48-24 N+34 p+d^{2}(1+4 p)+d(25-12 N+26 p)\right)\right) \\
&+H_{6} 2(N-p)\left(\alpha_{2}-\alpha_{1}\right)(2+d)(6-3 N+(4+d) p) \\
& Q_{4}=H_{0}\left(3+\alpha_{2}(d+1)\right)(2-N+p)(N-p) \\
&+H_{2}\left(3 \alpha_{1}-6(2+d)-\alpha_{2}\left(9+7 d+d^{2}\right)\right)(2-N+p)(N-p) \\
&+H_{4}(2+d)\left(-6 \alpha_{1}+\left(1+2 \alpha_{2}\right)(4+d)\right)(2-N+p)(N-p) \\
&+H_{6}\left(\alpha_{1}-\alpha_{2}\right)\left(8+6 d+d^{2}\right)(2-N+p)(N-p), \\
& \quad
\end{aligned}
$$


where $H_{i}$ are the functions

$$
\begin{aligned}
& H_{0}={ }_{2} F_{1}(1,1 / 2, d / 2,-\chi) \\
& H_{2}={ }_{2} F_{1}(1,3 / 2, d / 2+1,-\chi) / d \\
& H_{4}={ }_{2} F_{1}(1,5 / 2, d / 2+2,-\chi) \frac{3}{d(d+2)} \\
& H_{6}={ }_{2} F_{1}(1,7 / 2, d / 2+3,-\chi) \frac{15}{d(d+2)(d+4)} .
\end{aligned}
$$

Here, ${ }_{2} F_{1}$ is the Gauss hypergeometric function. The anomalous dimensions $\gamma_{[N, p]\left[N, p^{\prime}\right]}$ are

$$
\begin{aligned}
\gamma_{[N, p][N, p-2]} & =-\frac{g C_{d}}{4\left(d^{2}-1\right)} Q_{1}, & \gamma_{[N, p][N, p]} & =-\frac{g C_{d}}{4\left(d^{2}-1\right)} Q_{2}, \\
\gamma_{[N, p][N, p+2]} & =-\frac{g C_{d}}{4\left(d^{2}-1\right)} Q_{3}, & \gamma_{[N, p][N, p+4]} & =-\frac{g C_{d}}{4\left(d^{2}-1\right)} Q_{4},
\end{aligned}
$$

and the matrix of critical dimensions (32) is

$$
\Delta_{[N, p]\left[N, p^{\prime}\right]}=-N\left(1-\frac{\epsilon}{2}\right) \delta_{p p^{\prime}}+\gamma_{[N, p]\left[N, p^{\prime}\right]}^{*},
$$

where the asterisk stands for the value at the fixed point (27), (28). This represents the critical dimensions of the composite operators [38) at the first order in $\epsilon$. It should to be stressed that in contrast to the value of the fixed point (27), (28), which has no higher order corrections, the expressions for anomalous dimensions (39) have nonvanishing corrections of order $g^{2}$ and higher.

The critical dimensions are given by the eigenvalues of the matrix (40). As was already discussed in Ref. 13] in the limiting isotropic case $\left(\alpha_{1}=\alpha_{2}=0\right)$ this matrix becomes triangular, i.e., the eigenvalues are simply equal to the diagonal elements $\Delta[N, p] \equiv \Delta_{[N, p][N, p]}$.

Since our result for the anomalous dimensions is the same as in Ref. [13] for the admixture of a passive scalar, all conclusions about the hierarchical behavior of the critical dimensions of the composite operators are also valid in the analysis of the present model. Nevertheless, the inertial-range asymptotic behavior of the structure functions in these two problems is completely different, because, first, in the scalar problem single-point products of the scalar are not renormalized, while in the vector problem they are, and, second, the leading contribution to the OPE is given by the products of derivatives of the scalar, whereas in the vector problem products of the field components themselves yield the leading contribution.

In Ref. 13] the behavior of the critical dimensions $\Delta[N, p]$ for $N=2,3,4,5$, and 6 was numerically studied. The main conclusion is that the dimensions $\Delta_{N}$ remain negative in anisotropic case and decrease monotonically as $N$ increases for odd and even values of $N$ separately.

In present paper we concentrate our attention on the investigation of the composite operators (38) for relatively large values of $N$, namely we will analyze cases with $N=10,11,20,21,30,31,40,41,50$, and 51 . Our aim has been to find out whether hierarchies which hold for small values of $N$ remain valid for significantly larger values of $N$, and the answer turned out to be in the affirmative.

In Ref. [13] several hypothetically possible structures of the matrix of critical dimensions (40) were discussed. In particular, the possibility that the matrix (40) for some $\alpha_{1}$ and $\alpha_{2}$ would have a pair of complex conjugate eigenvalues $\Delta=\operatorname{Re} \Delta \pm i \operatorname{Im} \Delta$ cannot be excluded a priori. In this case, the small-scale behavior of the scaling functions would have oscillating terms of the form

$$
\left(\frac{r}{r_{l}}\right)^{\operatorname{Re} \Delta}\left\{C_{1} \cos \left[(\operatorname{Im} \Delta) r / r_{l}\right]+C_{2} \sin \left[(\operatorname{Im} \Delta) r / r_{l}\right]\right\},
$$

with some real constants $C_{1}, C_{2}$.

Another, in general, conceivable structure of the matrix (40) is related to the situation when it cannot be diagonalized but only reduced to the Jordan normal form. In this case, the corresponding contribution to the scaling function would involve a logarithmic correction to the power-like behavior, viz.

$$
\left(\frac{r}{r_{l}}\right)^{\Delta}\left[C_{1} \ln \left(r / r_{l}\right)+C_{2}\right]
$$

where $\Delta$ is the eigenvalue related to the Jordan cell. 

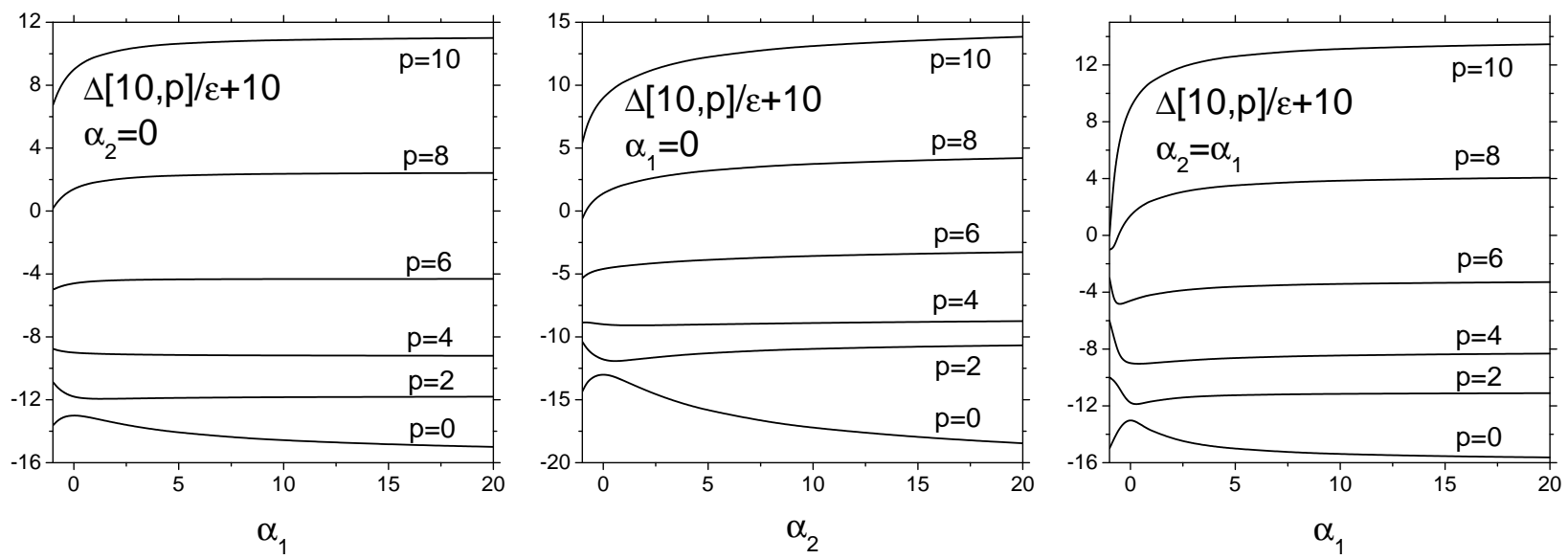

FIG. 2: Behavior of the critical dimension $\Delta[10, p] / \epsilon$ for space dimension $d=3$ and for representative values of $p$ as functions of anisotropy parameters $\alpha_{1}$ and $\alpha_{2}$.
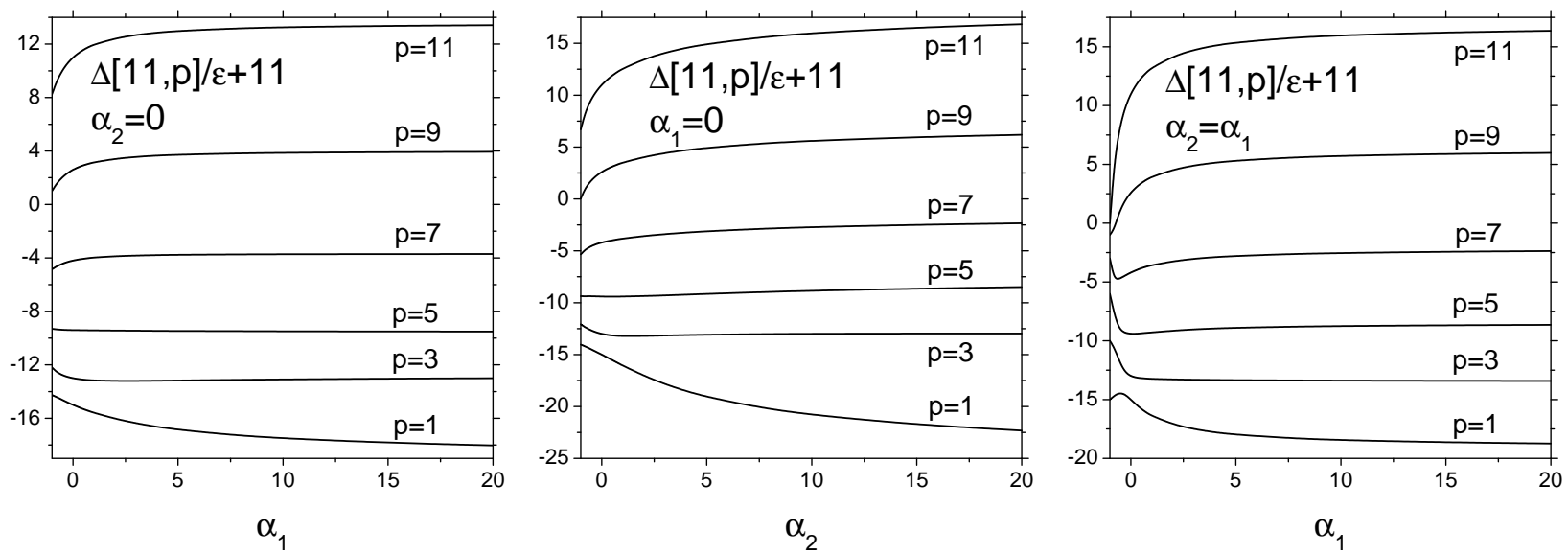

FIG. 3: Behavior of the critical dimension $\Delta[11, p] / \epsilon$ for space dimension $d=3$ and for representative values of $p$ as functions of anisotropy parameters $\alpha_{1}$ and $\alpha_{2}$.

In Figs. [2 7] behavior of the eigenvalues of the matrix of critical dimensions $\Delta[N, p]$ for relatively large values of the $N$ are shown. It can be seen that only real eigenvalues exist in all cases, and also their hierarchical behavior discussed in Ref. 13 is conserved. At first sight the curves for $p=0$ and $p=2$ in the even case and the curves for $p=1$ and $p=3$ in the odd case in Figs. 47 appear to be crossing at the point $\alpha_{1}=\alpha_{2}=0$ but in fact the curves are only visually running very near together at that point which is a mathematical consequence of the formulas for critical dimensions in the infinitesimal limit $\alpha_{1} \rightarrow 0$ and $\alpha_{2} \rightarrow 0$. 



FIG. 4: Behavior of the critical dimension $\Delta[30, p] / \epsilon$ for space dimension $d=3$ and for representative values of $p$ as functions of anisotropy parameters $\alpha_{1}$ and $\alpha_{2}$.
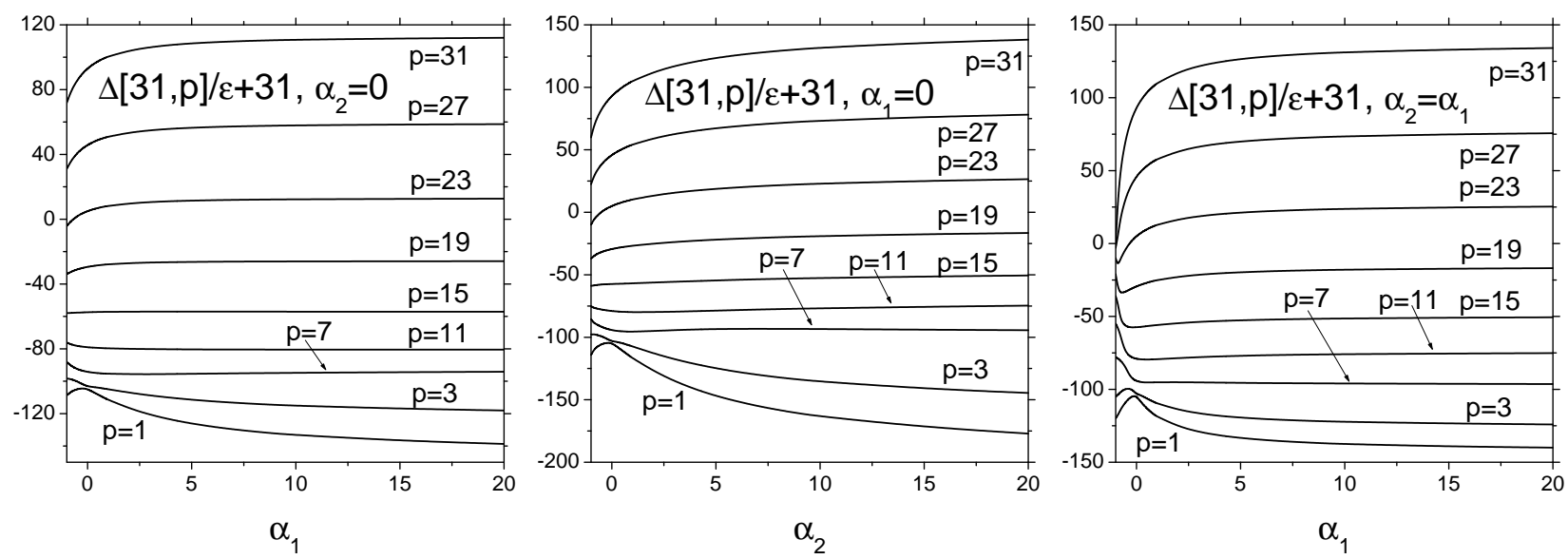

FIG. 5: Behavior of the critical dimension $\Delta[31, p] / \epsilon$ for space dimension $d=3$ and for representative values of $p$ as functions of anisotropy parameters $\alpha_{1}$ and $\alpha_{2}$.

\section{CONCLUSIONS}

In this paper we have analyzed asymptotic behavior of the structure functions $S_{N}$ of passively advected vector field with small-scale anisotropy. To this end field-theoretic renormalization group and the operator-product expansion have been used in a minimal-subtraction scheme of analytic renormalization.

It is shown that the leading-order asymptotic behavior of the structure functions is determined by the isotropic sector of the velocity field. At the leading order in the inertial interval all the structure functions are flat, i.e., independent of the separation distance, with powerlike corrections (with real positive exponents) effected by the small-scale anisotropy. We have calculated numerically the anomalous correction exponents up to order $N=51$ to explore possible oscillatory modulation or logarithmic corrections to the leading powerlike asymptotics, but have 



FIG. 6: Behavior of the critical dimension $\Delta[50, p] / \epsilon$ for space dimension $d=3$ and for representative values of $p$ as functions of anisotropy parameters $\alpha_{1}$ and $\alpha_{2}$.
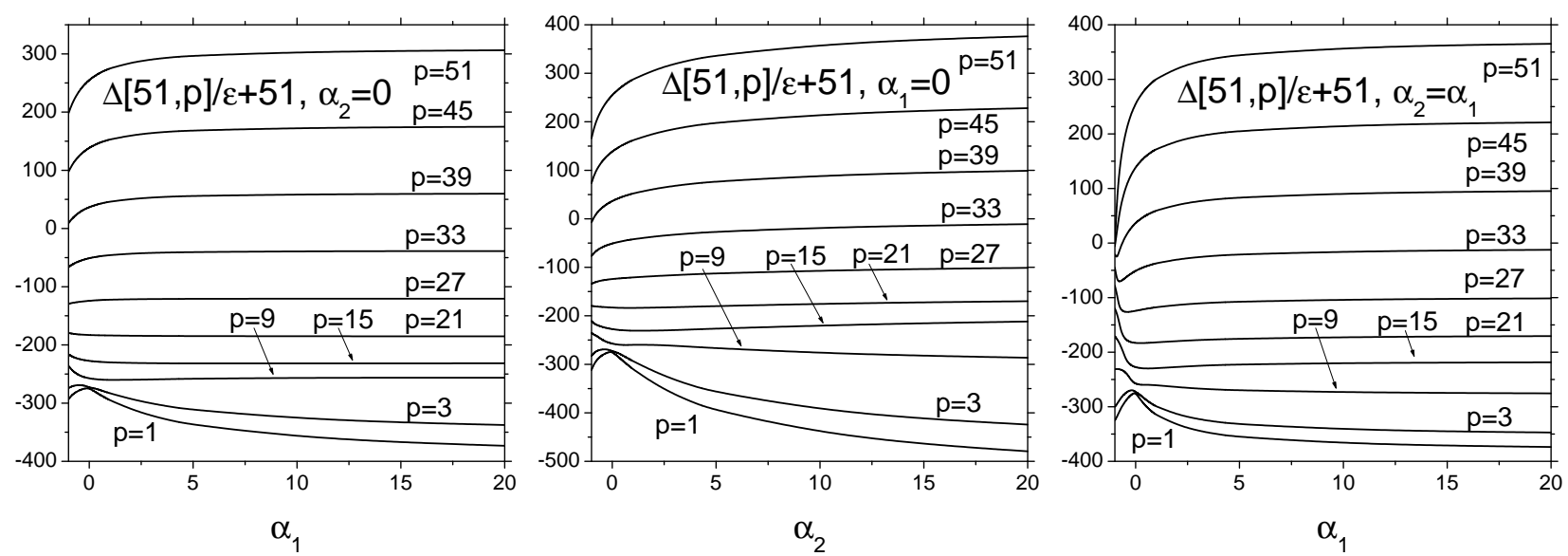

FIG. 7: Behavior of the critical dimension $\Delta[51, p] / \epsilon$ for space dimension $d=3$ and for representative values of $p$ as functions of anisotropy parameters $\alpha_{1}$ and $\alpha_{2}$.

found no sign of this kind of behavior: all calculated corrections have had purely powerlike behavior. Our results show that the exponents of the powerlike corrections tend to grow with increasing relative impact of the anisotropy.

From the renormalization-group point of view the present model of passively advected vector field in the presence of strong anisotropy has turned out to be very similar to that of passively advected scalar field [13]. In particular, the $\beta$ functions and the one-loop contributions to renormalization matrices of relevant composite operators are the same. Since in the published analysis of the scalar problem [13] there were some misprints, we have also presented corrected complete results of the calculation of the renormalization matrices.

However, physically the two models differ significantly: instead of the anomalous powerlike growth of the structure functions of the scalar problem in the inertial range, in the present vector case the leading asymptotic term turned out to be constant with powerlike corrections due to the small-scale anisotropy. Thus, for the passively advected 
vector field a much stronger departure from the Kolmogorov-like scaling than in the case of passively advected scalar is predicted.

\section{Acknowledgments}

M. H. gratefully acknowledges the hospitality of the N. N. Bogoliubov Laboratory of Theoretical Physics at JINR, Dubna, the University of Genova, Italy, and the Department of Physical Sciences of the University of Helsinki, Finland. J. H. thanks the Institute for Experimental Physics of the Slovak Academy of Sciences in Košice for hospitality.

This work was supported in part by the Slovak Academy of Sciences (VEGA Grant No. 3211), by the Academy of Finland (Grant No. 203121), and by the common grant CNR (Italy) and SAS (Slovakia).

[1] A.S. Monin and A.M. Yaglom, Statistical Fluid Mechanics (MIT Press, Cambridge, MA, 1975), Vol. 2.

[2] S.A. Orszag, Lectures on the Statistical Theory of Turbulence, in Fluid Dynamics (Les Houches Lectures) edited by R. Balian and J.L. Peube (Gordon \& Breach, London, 1973), p. 235.

[3] U. Frisch, Turbulence: the legacy of A. N. Kolmogorov (Cambridge University Press, Cambridge, 1995).

[4] R.A. Antonia, B.R. Satyaprakash, and A.K.M.F. Hussain, J. Fluid Mech. 119, 55 (1982);

F. Anselmet, Y. Gagne, E. Hopfinger, and R.A. Antonia, J. Fliud Mech. 140, 63 (1984);

C. Meneveau, and K.R. Sreenivasan, Phys. Rev. A 41, 2246 (1990);

V.R. Kuznetsov and V.A. Sabel'nikov, Turbulence and Combustion (Hemisphere Publishing, New York, 1990); M.S. Borgas, Phys. Fluids A 4, 2055 (1992).

[5] J. Zinn-Justin, Quantum Field Theory and Critical Phenomena (Clarendon, Oxford, 1989).

[6] A.N. Vasil'ev, The Field Theoretic Renormalization Group in Critical Behavior Theory and Stochastic Dynamics (Chapman\&Hall/CRC,Boca Raton, 2004)

[7] L.Ts. Adzhemyan, N.V. Antonov, and A.N. Vasiliev, Usp. Fiz. Nauk, 166, 1257 (1996) [Phys. Usp. 39, 1193 (1996)].

[8] L.Ts. Adzhemyan, N.V. Antonov, and A.N. Vasiliev, The Field Theoretic Renormalization Group in Fully Developed Turbulence (Gordon and Breach, London, 1999).

[9] C. De Dominicis, P.C. Martin, Phys. Rev. A 19, 419 (1979).

[10] H.W. Wyld, Ann. Phys. 14, 143 (1961).

[11] R.H. Kraichnan, Phys. Rev. Lett. 72, 1016 (1994).

[12] L.Ts. Adzhemyan, N.V. Antonov, A. Mazzino, P. Muratore-Ginanneschi, A.V. Runov, Europhys. Lett. 55, 801 (2001); L.Ts. Adzhemyan, N.V. Antonov, A.V. Runov, Phys. Rev. E 64,046310 (2001);

N.V. Antonov, J. Honkonen, A. Mazzino, P. Muratore-Ginanneschi, Phys. Rev. E 62, 5891 (2000);

N.V. Antonov, A. Lanotte, A. Mazzino, Phys. Rev. E 61, 6586 (2000);

A. Lanotte, A. Mazzino, Phys. Rev. E 60, 3483 (1999).

[13] L.Ts. Adzhemyan, N.V. Antonov, M. Hnatich, S.V. Novikov, Phys. Rev. E 63, 016309 (2001).

[14] L.Ts. Adzhemyan, N.V. Antonov, A.N. Vasil'ev, Phys. Rev. E 58, 1823 (1998).

[15] L.Ts. Adzhemyan, N.V. Antonov, Phys. Rev. E 58, 7381 (1998).

[16] N.V. Antonov, Phys. Rev. E 60, 6691 (1999);

N.V. Antonov, Physica D 144, 370 (2000).

[17] I.S. Gradshtejn and I.M. Ryzhik, Tables of Integrals, Series and Products (Academic, New York, 1965).

[18] M. Hnatich, M. Jurcisin, A. Mazino, and S. Sprinc, Acta Physica Slovaca 52, 559 (2002).

[19] L.Ts. Adzhemyan, N.V. Antonov, V.A. Barinov, Yu.S. Kabrits, and A.N. Vasiliev, Phys. Rev. E 63, 025303(R) (2001).

[20] L.Ts. Adzhemyan, N.V. Antonov, V.A. Barinov, Yu.S. Kabrits, and A.N. Vasiliev, Phys. Rev. E 64, 056306 (2001).

[21] R. Rubinstein and J.M. Barton, Phys. Fluids 30, 2986 (1987);

D. Carati and L. Brenig, Phys. Rev. A 40, 5193 (1989);

L.Ts. Adzhemyan, M. Hnatich, D. Horvath, and M. Stehlik, Int. J. Mod. Phys. B 9, 3401 (1995);

T.L. Kim and A.V. Serdukov, Theor. Math. Phys. 105, 412 (1995);

J. Buša, M. Hnatich, J. Honkonen, and D. Horvath, Phys. Rev. E 55, 381 (1997);

N.V. Antonov, A.V. Runov, Teor. Mat. Fiz. 112, 417 (1997);

M. Hnatich, E. Jonyova, M. Jurcisin, and M. Stehlik, Phys. Rev. E 64, 016312 (2001);

J. Busa, M. Hnatich, E. Jurcisinova, M. Jurcisin, and M. Stehlik, Acta Physica Slovaca 52, 547 (2002).

[22] P. C. Martin, E. D. Siggia and H. A. Rose, Phys. Rev. A 8, 423 (1973);

H.K. Janssen, Z. Phys. B 23, 377 (1976);

R. Bausch, H. K. Janssen and H. Wagner, Z. Physik B 24, 113 (1976);

C. DeDominicis, J. Phys. (Paris), Colloq. 37, C1-247 (1976). 